\title{
Trk Agonist Drugs Rescue Noise-Induced Hidden Hearing Loss
}

Katharine A. Fernandez ${ }^{1,2, \#}$, Takahisa Watabe ${ }^{1,2, \#}$, Mingjie Tong ${ }^{1,2}$, Xiankai Meng ${ }^{1,2}$, Kohsuke Tani ${ }^{1,2}$, Sharon G. Kujawa ${ }^{1,2,3, *}$, Albert S. B. Edge ${ }^{1,2,3,4, *}$

\# These authors contributed equally.

${ }^{1}$ Department of Otolaryngology, Harvard Medical School, Boston, Massachusetts 02115

${ }^{2}$ Eaton-Peabody Laboratories, Massachusetts Eye and Ear, Boston, Massachusetts 02114

${ }^{3}$ Program in Speech and Hearing Bioscience and Technology, Harvard Medical School, Boston, Massachusetts 02115

${ }^{4}$ Harvard Stem Cell Institute, Cambridge, Massachusetts 02138

*Correspondence should be addressed to:

Albert Edge

Massachusetts Eye and Ear

243 Charles St.

Boston, MA 02114

Sharon Kujawa

Massachusetts Eye and Ear

243 Charles St.

Boston, MA 02114 


\begin{abstract}
TrkB agonist drugs are shown here to have a significant effect on the regeneration of afferent cochlear synapses after noise-induced synaptopathy. The effects were consistent with regeneration of cochlear synapses that we observed in vitro after synaptic loss due to kainic acid-induced glutamate toxicity and were elicited by administration of TrkB agonists, amitriptyline and 7,8- dihydroxyflavone, directly into the cochlea via the posterior semicircular canal $48 \mathrm{~h}$ after exposure to noise. Synaptic counts at the inner hair cell and wave 1 amplitudes in the ABR were partially restored 2 weeks after drug treatment. Effects of amitriptyline on wave 1 amplitude and afferent auditory synapse numbers in noise-exposed ears after systemic (as opposed to local) delivery were profound and long-lasting; synapses in the treated animals remained intact one year after the treatment. However, the effect of systemically delivered amitriptyline on synaptic rescue was dependent on dose and the time window of administration: it was only effective when given before noise exposure at the highest injected dose. The long-lasting effect and the efficacy of post-exposure treatment indicate a potential broad application for the treatment of synaptopathy, which often goes undetected until well after the original damaging exposure(s).
\end{abstract}




\section{Introduction}

Sensorineural hearing loss results from pathology of the inner ear and its primary cause can reside in sensory hair cells or afferent neurons (Kujawa and Liberman, 2006, 2009; Nadol, 1997; Ruel et al., 2007; Sergeyenko et al., 2013; Wang et al., 2002; Wong and Ryan, 2015). However, work in animal models of noise- and age-related hearing loss has revealed that loss of synapses between the two is commonly the earliest finding (Kujawa and Liberman, 2009). This type of damage has been referred to as 'hidden hearing loss' because its presence is not captured by the threshold audiogram, the standard clinical assay of hearing loss. Although synaptic loss is not revealed by threshold measures until near total, it can be detected as a decline in the suprathreshold amplitudes of neural responses (Fernandez et al., 2020; Kujawa and Liberman, 2009; Sergeyenko et al., 2013).

Neurotrophins have been tested for activity in ameliorating noise- and toxininduced damage to the neurons of the inner ear (Ramekers et al., 2015; Ruel et al., 2007; Shepherd et al., 2005; Shinohara et al., 2002; Wise et al., 2005). These molecules have numerous functions in neurons, including support for neural survival, outgrowth of neurites, and both guidance and synapse formation. While neurotrophins have been extensively tested as therapeutic agents, the difficulty in targeting therapeutic levels of proteins to the brain or peripheral nerves has hampered their use (Poduslo and Curran, 1996). In contrast, small molecules can reach target tissues in the nervous system, with demonstrated protective and regenerative effects (Jang et al., 2009).

Here, we examined the potential of amitriptyline (AT) and 7,8-dihydroxyflavone (DHF), structurally unrelated small molecule agonists of the TrkB receptor (Shibata et 
al., 2007; Yu et al., 2012; Yu et al., 2013), for the protection or regeneration of cochlear neural function caused by synaptopathic noise exposure. Using parallel in vitro and in vivo approaches, we characterized protection or rescue from cochlear synapse loss/deafferentation in the mouse cochlea. We found that TrkB agonists were strikingly effective in protection and restoration of synapses and neural function in noise-exposed ears.

\section{Materials and Methods}

$\underline{\text { Animals }}$ - Mice $(\mathrm{CBA} / \mathrm{CaJ})$ were born and raised in our acoustically monitored animal care facility (Sergeyenko et al., 2013) from breeders purchased from Jackson Laboratory (Bar Harbor, Maine). All experiments were conducted in accordance with the Public Health Service policy on Humane Care and Use of Laboratory Animals.

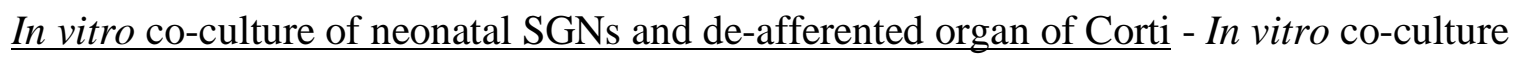
of SGN and de-afferented organ of Corti was performed according to the method described previously (Parker et al., 2010; Tong et al., 2013). Briefly, isolated SGNs were treated with $0.25 \%$ trypsin for $15 \mathrm{~min}$ at $37^{\circ} \mathrm{C}$. After neutralization of the trypsin with $10 \%$ FBS in DMEM, neurons were collected by centrifugation, triturated to a single-cell suspension and co-cultured with the de-afferented organ of Corti. The organ was dissected by removal of the membranous labyrinth, the stria vascularis and spiral ligament. Reissner's membrane and the tectorial membrane were removed, and the organ was cultured in a well on a cover glass coated with $1: 1$ poly-L-ornithine $(0.01 \%$; SigmaMillipore, USA) and laminin (50 $\mu \mathrm{g} / \mathrm{ml}$; BD Biosciences, USA). Explants were cultured 
in N2 and B27- supplemented DMEM, 1\% HEPES solution (Millipore Sigma, USA), 1:1000 ampicillin, $1: 300$ fungizone at $37^{\circ} \mathrm{C}, 6.5 \% \mathrm{CO}$. Amitriptyline (AT; $0.5 \mu \mathrm{M}$; Sigma, USA) was added at the beginning of the co-culture and cultures were stopped after 6 days. The number of innervated inner hair cells at the end of the incubation was quantified (Brugeaud et al., 2014; Parker et al., 2010).

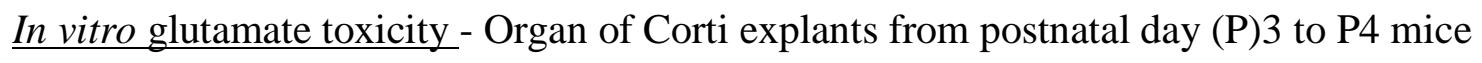
of both sexes, after $24 \mathrm{~h}$ in culture, were exposed to a solution of $0.4 \mathrm{mM}$ kainic acid (Abcam, USA) diluted in culture medium. After a 1-h kainic acid treatment, the explants were washed 6 times with culture medium and maintained for $24 \mathrm{~h}$ in $80 \mu \mathrm{l}$ of culture medium alone or containing AT $(0.1 \mu \mathrm{M})$ or 7,8-dihydroxyflavone (DHF; $0.5 \mu \mathrm{M}$;

Sigma, USA). The cultures were then fixed and prepared for immunofluorescence.

Acoustic overexposure - Noise exposure (8-16 kHz octave-band noise, $100 \mathrm{~dB}$ SPL, $2 \mathrm{~h}$ ) was conducted on awake, adult (16-wk) mice as described previously (Kujawa and Liberman, 2009). The noise was created by a waveform generator (model WGI; TuckerDavis Technologies), filtered (8-16 kHz, >60 dB/octave slope; Frequency Devices), amplified (D-75 power amplifier; Crown Audio) and delivered (compression driver; JBL) through an exponential horn into a tabletop reverberant exposure chamber. Mice were placed in a subdivided cage suspended directly beneath the acoustic horn of the sounddelivery speaker. Prior to each exposure, the noise was calibrated to the target $100 \mathrm{~dB}$ SPL using a quarter-inch condenser microphone (variability $<1 \mathrm{~dB}$ across subdivisions). 
Drugs and delivery - To optimize drug access to the cochlea, direct infusion of drug into the posterior semicircular canal was performed. For these experiments, awake animals were noise-exposed and held for $48 \mathrm{~h}$. They were then anesthetized (ketamine $100 \mathrm{mg} / \mathrm{kg}$ and xylazine $10 \mathrm{mg} / \mathrm{kg}$, IP). A small hole was made in the posterior semicircular canal and $500 \mathrm{nl}$ of drug suspension (25 mM AT in artificial perilymph; or $5 \mathrm{mM}$ DHF in $10 \%$ DMSO in artificial perilymph; or artificial perilymph without drug) was injected at a rate of $91 \mathrm{nl} / \mathrm{min}$. At the end of the infusion, the needle was left in place for $5 \mathrm{~min}$, after which the surgical defect was repaired. Animals were held for 2 wks before testing and processing.

To assess effects with less invasive, systemic delivery of drugs, we focused on AT and animals received intraperitoneal (IP) injections of AT in saline or an equal volume of the saline vehicle alone according to the following treatment groups: 1) AT $(12.5,25$ or $50 \mathrm{mg} / \mathrm{kg})$ or saline once daily for 5-9 days with noise exposure $6 \mathrm{~h}$ after the $3^{\text {rd }}$ day of treatment; 2) AT (50 mg/kg) or saline once, $6 \mathrm{~h}$ prior to noise exposure; 3 ) AT $(50 \mathrm{mg} / \mathrm{kg})$ or saline once, $3 \mathrm{~d}$ after noise exposure; 4) AT (50 mg/kg) or saline once daily for 9 days beginning $3 \mathrm{~d}$ after noise exposure. Groups of mice were evaluated $24 \mathrm{~h}$ after noise to assess acute losses, 2 wks after noise, when threshold recovery for this exposure is complete and synapse loss has stabilized (Kujawa and Liberman 2009) or were held for one year post exposure to examine long-term effects.

Cochlear function tests - Distortion product otoacoustic emissions (DPOAEs) and auditory brainstem responses (ABRs) were recorded from anesthetized mice (ketamine $100 \mathrm{mg} / \mathrm{kg}$ and xylazine $10 \mathrm{mg} / \mathrm{kg}, \mathrm{IP}$ ) in a $37^{\circ} \mathrm{C}$, acoustically- and electrically-shielded 
chamber. A small incision made in the cartilaginous portion of the external ear canal provided a clear view to evaluate the health of the tympanic membrane and optimized acoustic system placement. Physiologic responses were stimulated and responses were collected using a 24-bit National Instruments PXI-based system controlled by custom LabView-based software. The acoustic system consisted of two miniature dynamic earphones (CDMF15008-03A; CUI) and a condenser microphone (FG-23329-PO7; Knowles) coupled to a probe tube.

Outer hair cell (OHC)-based DPOAEs were recorded in response to two primary tones, $\mathrm{f} 1$ and $\mathrm{f} 2$, with $\mathrm{f} 2$ equal to the frequencies used in ABR testing, $\mathrm{f} 2 / \mathrm{f} 1=1.2$ and $\mathrm{L} 2$ $=\mathrm{L} 1-10 \mathrm{~dB}$. For each frequency pair, DPOAE responses at 2f1-f2 were recorded, averaged, and analyzed as a function of level (L2: below threshold to $80 \mathrm{~dB}$ SPL) from sound pressure measurements in the ear canal. Threshold was defined as the L2 stimulus level required to generate a DPOAE amplitude of $-5 \mathrm{~dB}$ SPL.

Neural-based ABRs were measured in response to tone pips (5.6 to $45.2 \mathrm{kHz} ; 0.5$ ms rise-fall, 5 ms duration, 30/s, alternating polarity) using subdermal electrodes placed at the vertex, ventrolateral to the pinna, and at the base of the tail (ground). Stimuli were delivered at subthreshold levels to $90 \mathrm{~dB}$ SPL in $5 \mathrm{~dB}$ steps. Amplified (10,000X) responses were filtered $(0.3-3 \mathrm{kHz})$, digitized, and averaged for each of the frequencylevel combinations (1024 samples/level). Threshold was identified via visual inspection of stored waveforms as the lowest stimulus level needed to elicit a repeatable wave I response. 
$\underline{\text { Immunohistochemistry and quantification of afferent synapses - Immediately following }}$ the physiologic testing, anesthetized mice were transcardially perfused with $4 \%$ paraformaldehyde in $0.1 \mathrm{M}$ phosphate buffer with additional perfusion through the cochlear scalae. Cochleas were post fixed in $4 \%$ paraformaldehyde for $1 \mathrm{~h}$ then decalcified (0.12 M EDTA) for up to $48 \mathrm{~h}$. Microdissected pieces were placed in a blocking buffer (PBS with 5\% normal horse serum and 0.3\% Triton X-100) for $1 \mathrm{~h}$ at room temperature followed by overnight incubation at $37^{\circ} \mathrm{C}$ in antibodies to the following: (1) C-terminal binding protein 2 (mouse anti-CtBP2; BD Biosciences, used at 1:200). (2) myosin-VIIa (rabbit anti-myosin-VIIa; Proteus Biosciences; used at 1:200), and (3) GluA2 (mouse anti-glutamate receptor 2; Millipore; used at 1:2000). Cochlear pieces were incubated in species appropriate secondary antibodies coupled to Alexa Fluors in the red, blue, and green channels for $2 \mathrm{~h}$ at $37^{\circ} \mathrm{C}$.

Cochlear piece lengths, collected and compiled using ImageJ software, were used to create a cochlear frequency map to localize cochlear structures to specific frequency regions (Muller et al., 2005). Confocal z-stacks were obtained (Leica TCS SP5) using a high-resolution, glycerin-immersion objective (63x, 1.3 N.A.) and a 3.17x digital zoom, 1024 x 512 raster. Two adjacent stacks ( $0.25 \mu \mathrm{m}$ step size) were imaged for each targeted frequency region, each image spanning $78 \mu \mathrm{m}$ of cochlear length. Confocal image stacks were ported to image-processing software (AMIRA; VISAGE Imaging) where inner hair cells (IHCs) and synapses were quantified as previously described (Fernandez et al., 2015; Sergeyenko et al., 2013). Synaptic associations between presynaptic IHC ribbons and post-synaptic glutamate receptors (synapses) were determined with custom software that computed and displayed the $x-y$ projections of the 
voxel space within 1 um of the center of each ribbon identified by Amira analysis (Fernandez et al., 2015).

Immunohistochemistry and quantification of regenerated afferent synapses in the in vitro assays were performed by the same methods (Tong et al., 2013). Following fixation and permeabilization for $1 \mathrm{~h}$, cultures were incubated with primary antibodies, anti-CtBP2 (mouse monoclonal IgG1; BD Biosciences, USA, used at 1:1,000), anti-PSD95 (mouse monoclonal IgG2a; NeuroMab, USA, used at 1:1,000), and anti-neurofilament (chicken antibody to neurofilament-H; Millipore, USA, used at 1:2,500) overnight at $4^{\circ}$ C. After rinsing three times for 10 min with 0.01 M PBS, pH $\square$ 7.4. They were incubated with secondary antibodies: cyanine-5-conjugated goat anti-mouse IgG1 (Caltad Laboratories), biotin-conjugated Fluor goat anti-mouse IgG2a (Caltad Laboratories), Alexa Fluor 568-Streptavidin (Molecular Probes) and Alexa Fluor 488 goat anti-chicken (Molecular Probes) for $2 \mathrm{~h}$ at room temperature. Newly generated afferent synapses were identified by triple labeling of CtBP2, PSD-95 and neurofilament.

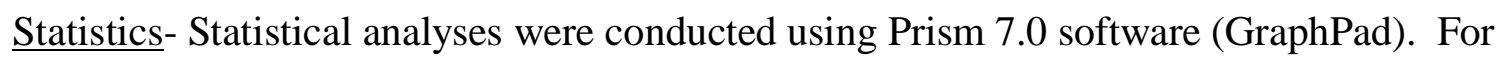
analyses on adult mice, a one-way or two-way analysis of variance (ANOVA) was used, followed by Bonferroni correction for multiple comparisons. For analyses on neonatal mice, a two-tail, unpaired Students' $t$-test was used. For all analyses, an alpha of $<0.05$ was considered significant.

\section{Results}

\section{$\underline{\mathrm{AT}}$ and DHF increased cochlear synaptic regeneration in vitro}


To assess synapse formation in a culture system, in which SGNs were co-cultured with de-afferented organ of Corti (Martinez-Monedero et al., 2006; Tong et al., 2013), the co-cultures were treated with $0.5 \mu \mathrm{M}$ AT or maintained as controls. Six days later, immunohistochemistry demonstrated that cochlear afferent synapses had regenerated in the cultures (Fig. 1A and B). Quantitative measurement indicated a significant increase in synaptic regeneration in the AT treated cultures (Fig. 1C, $* \mathrm{p}<0.05$ ).

We adapted a rat model of excitatory cochlear synaptopathy (Wang and Green, 2011) to the mouse explants to assess the regenerative effect of the drug on cochlear synapses. In this model, cochlear explants, consisting of hair cells and attached SGNs, are exposed to selective ionotropic glutamate receptor agonist, kainic acid, to mimic glutamate toxicity, the excessive release of glutamate into the synaptic cleft with attendant damage to the terminal processes of SGNs (Pujol et al., 1985; Pujol and Puel, 1999). Synapses were detected by the occurrence of CtBP2-expressing synaptic ribbons and PSD95-positive post-synaptic densities. Damage was extensive after $1 \mathrm{~h}$ of treatment with kainic acid, and a small amount of reinnervation of hair cells by the peripheral processes of the neurons was apparent after $24 \mathrm{~h}$ in the absence of drugs (Fig. 1D). Addition of $0.1 \mu \mathrm{M}$ AT (Fig. 1E) or $0.5 \mu \mathrm{M}$ DHF (Fig. 1F) to the in vitro synaptogenesis assay resulted in a significant increase in juxtaposed ribbons and PSD95-positive neural endings relative to untreated samples.

\section{$\underline{\text { AT rescued synapses and auditory function in vivo }}$}

Prior studies in adult CBA/CaJ mice have demonstrated that exposure to a $2 \mathrm{~h}$, $100 \mathrm{~dB}$ SPL octave band noise $(8-16 \mathrm{kHz})$ results in a large, but reversible threshold 
shift, as evidenced by DPOAE and ABR wave-I measures. The exposure causes no acute loss of inner or outer hair cells but initiates an immediate and largely permanent loss of synapses between auditory nerve terminals and cochlear IHCs in mid to high frequency locations along the basilar membrane. This is accompanied by persistent declines in suprathreshold amplitudes of neural, but not OHC responses (Fernandez et al., 2015; Kujawa and Liberman, 2009, 2015). Here, we aimed to determine whether Trk agonist treatment could ameliorate some of the irreversible damage and reduce neural function caused by this noise exposure.

We reasoned that direct administration of the TrkB agonists to perilymph would be the best route for administration of the drugs in vivo. We delivered AT or DHF or vehicle alone into perilymph via posterior semicircular canal injection $48 \mathrm{~h}$ after noise exposure. When tested $24 \mathrm{~h}$ after exposure (before drug treatment), animals displayed similar ABR wave 1 threshold elevations at test frequencies above the noise band (21-45 kHz, Fig. 2A). Thresholds recovered to within about 5 dB by 2 wks (Fig. 2B). Results are consistent with our previous reports (Kujawa and Liberman 2009; Fernandez et al., 2015) and demonstrate, further, that the drug treatments did not alter threshold recovery after noise.

Both AT- and DHF-treated mice demonstrated significantly greater neural response amplitudes than mice treated with the vehicle alone when assessed at the 2 wk post-exposure time point (2-way $\left.\operatorname{ANOVA}, \mathrm{F}_{(2,130)}=4.636, \mathrm{p}=0.0114\right)$. In basal cochlear regions of maximum noise injury, vehicle-treated mice showed $\sim 45 \%$ wave 1 amplitude reductions compared to unexposed mice, whereas amplitudes recorded in AT- and DHFtreated animals recovered to $\sim 70 \%$ of unexposed controls (see for example $30 \mathrm{kHz}$, Fig. 
3A). Proportionately, and in the same cochlear region, AT- and DHF-treated mice demonstrated significantly greater synapse counts than mice treated with vehicle alone (2-way ANOVA, $\left.\mathrm{F}_{(2,72)}=8.182, \mathrm{p}=0.0006\right)$. Two weeks following exposure, synaptic declines of almost $50 \%$ were observed in the noise-damaged basal cochlea of vehicletreated mice, whereas counts recorded in AT- and DHF-treated animals recovered to 70$75 \%$ of unexposed controls.

\section{AT preserved synapses and auditory function in vivo when administered}

\section{systemically prior to noise-exposure}

Given the effects of directly administered drug, and the potential to give these drugs systemically due to their low risk safety profile, we next asked whether the same effects could be achieved by systemic delivery. We performed these experiments with AT.

In our initial experiments, mice were treated systemically with saline or AT (12.5, 25 , or $50 \mathrm{mg} / \mathrm{kg}$ ) before and after noise exposure. Subsets of animals were tested $24 \mathrm{~h}$ after exposure and displayed expected and similar threshold elevations for both DPOAEs and ABR wave I at test frequencies above the noise band. Thresholds recovered by 2 wks as shown previously for this exposure (Kujawa and Liberman 2009; Fernandez et al., 2015; Fernandez et al., 2020). DPOAE amplitudes at 2 wks showed essentially complete post-exposure recovery for all drug and saline treatment groups (Fig. 4a). Together with the DPOAE and ABR wave 1 threshold recovery, the finding is consistent with functionally intact $\mathrm{OHCs}$ and no effect of the drugs on their post-exposure recovery. 
In contrast, two weeks after noise, animals showed permanent ABR wave 1 amplitude decrements that varied with treatment and dose (Fig. 4B). At the highest dose of AT, $50 \mathrm{mg} / \mathrm{kg}$, response amplitudes averaged over $80-80 \mathrm{~dB}$ were significantly larger [2-way ANOVA, $\mathrm{F}_{(3,238)}=11.07, \mathrm{p}<0.0001$; Bonferroni multiple comparison significance at $17 \mathrm{kHz}(\mathrm{p}<0.03)$ and $30 \mathrm{kHz}(\mathrm{p}<0.001)]$ than those recorded for animals in the other groups. Synapse counts at cochlear regions spanning the frequency regions assessed physiologically also were greater in drug-treated animals (2-way ANOVA, $\mathrm{F}_{(1,116)}=18.90$, p<0.0001) (Fig. 4C). Vehicle-treated mice showed a maximum 50\% synapse loss relative to unexposed controls, whereas they were nearly normal for AT $50 \mathrm{mg} / \mathrm{kg}$-treated mice.

Subsequent experiments focused on the effective dose $(50 \mathrm{mg} / \mathrm{kg})$ and compared outcomes for treatments given only before or only after noise, as follows: 1) once, $6 \mathrm{~h}$ before noise, 2) once, $3 \mathrm{~d}$ after noise and 3) once daily for 9 days beginning 3 days after exposure.

Post-exposure threshold recovery by 2 wks was again unaltered by the treatments. However, wave 1 amplitudes and synapse counts at basal cochlear frequencies were sensitive to the timing of treatment relative to noise exposure; both were near normal when AT was given before (or before and after) noise, but declined to $\sim 50 \%$ when AT was administered only after noise (Figs. 5A, B). For animals receiving single dose prenoise AT, suprathreshold wave 1 amplitudes and IHC synapse counts at 2 wks were $\sim 50$ $60 \%$ larger than saline controls in the noise-damage region (17 and $30 \mathrm{kHz})$. However, when the same AT dose was delivered $6 \mathrm{~h}$ after noise, neither ABR wave-I amplitudes (Fig. 5A) nor synapse counts (Fig. 5B) were systematically different from those recorded for saline controls: Amplitudes: $F_{(1,58)}=3.742, p=0.0579$; Synapses: $F_{(1,112)}=0.06407$, 
$\mathrm{p}=0.8006$. Thus, AT showed potent protection but only at the highest dose $(50 \mathrm{mg} / \mathrm{kg})$, and a single injection at high concentration was as effective as repeated injections.

\section{$\underline{\text { AT preserved synapses and auditory function in vivo one-year after noise exposure }}$}

Prior work has documented gradually-progressive loss of synapses with aging, and acceleration of these losses with spread to more apical regions in animals receiving synaptopathic noise exposure as young adults (Sergeyenko et al., 2013; Fernandez et al., 2015). Here, subsets of animals from the initial experimental series (systemic AT 12, 25, $50 \mathrm{mg} / \mathrm{kg}$ ) were followed to 1 year post exposure to assess long-term effects of drug treatments on cochlear deafferentation. Shown in Figures 6A and B are DPOAE and ABR wave 1 amplitudes $(30 \mathrm{kHz})$ for these long-held groups. OHC-based DPOAEs are similar across treated groups, documenting minimal exacerbation of ongoing declines relative to age-only mice (Fernandez et al., 2015). Of note, the neuroprotective treatment effect of AT apparent at 2 wks (Fig. 4C) persists, with mice that received pre-noise AT $(50 \mathrm{mg} / \mathrm{kg})$ displaying significantly greater neural response amplitudes at $17 \mathrm{kHz}(\mathrm{p}<0.01)$ and $30 \mathrm{kHz}(\mathrm{p}<0.05)$ than vehicle-treated animals at the $1 \mathrm{yr}$ test time (2-way ANOVA, $\mathrm{F}_{(1,69)}=11.46, \mathrm{p}<0.01$ with Bonferroni multiple comparisons test). Indeed, with responses little different from never-exposed controls, these animals appear to have been fully protected from the noise-induced cochlear de-afferentation.

Support for this notion also can be found in the synapse counts from these mice (Fig. 6C). High-dose AT treated mice showed significantly greater $\left(\mathrm{F}_{(1,131)}=16.71\right.$, $\mathrm{p}<0.0001)$ synapse counts than vehicle-treated ears at $30 \mathrm{kHz}(\mathrm{p}<0.0001)$ where counts were consistent with age-matched, unexposed mice. Moreover, as documented 
previously, the damage region in aging vehicle-treated ears spread apically toward the previously unaffected region of $11 \mathrm{k} \mathrm{Hz}$, where synapse counts were now $20 \%$ greater in age-matched, AT-treated mice.

When compared to data collected for animals held 2 wks following noise exposure, wave 1 amplitude differences between saline and AT-treated mice were exaggerated at $1 \mathrm{yr}$, with vehicle-treated animals showing greater ongoing declines after noise. Of note, DPOAE amplitudes recorded from the same ears were comparable for all groups. Together, findings show that drug-related effects target synapses/neurons rather than OHCs, effects are longlasting, and the increasing separation between saline and AT $(50 \mathrm{mg} / \mathrm{kg})$ is not reflected at the level of the OHCs. Additionally, as synapse counts in saline-treated animals show results consistent with our previous reports in exposed, then aged mice (Fernandez et al., 2015), the significantly smaller age-progressive declines for AT-treated mice suggest that the drug also may have long-term protective effect against age-progressive de-afferentation.

\section{Discussion}

We have shown here that treatment with AT restores synapses between cochlear afferent neurons and sensory inner hair cells. In contrast to the ears of control (salinetreated, noise-exposed) animals, in which extensive noise-induced afferent synapse loss persists after thresholds recover, synapses were significantly protected by pre-exposure treatment and rescued by post-exposure treatment with AT, and cochlear neurons appeared functionally intact. The protective effect of pre-noise AT against cochlear deafferentation was long-lasting. The regenerative effect of the drug is particularly significant for clinical application, as exposure to noise, which may be a principal 
contributor to synaptopathy in the human population, can occur in varied environments with unpredictable timing and may be cumulative. The effect of AT in mitigating the synaptic loss and promoting synaptogenesis was also seen in vitro, providing support for a regenerative mechanism and pointing to TrkB receptor involvement.

Synaptopathy occurs as a primary consequence of noise exposure and can permanently reduce cochlear afferent response amplitudes in ears with functionally intact hair cells and normal thresholds (Kujawa and Liberman, 2009). Correlation between loss of amplitude and synaptic loss suggested a 'hidden hearing loss' in which audiograms (assays of threshold sensitivity) could recover to normal and the loss of synapses preceded the loss of hair cells and could act independently of it. However, even when thresholds recover, this cochlear deafferentation may compromise the fidelity of suprathreshold signal coding and moreover appears to be a harbinger of exaggerated declines in hearing function (Kujawa and Liberman, 2006, 2009). Indeed this type of mechanism for loss of neural response amplitude has now been shown to be important in the loss of cochlear function caused by aging as well as noise exposure (Sergeyenko et al., 2013).

The activity we find here for a Trk agonist is consistent with previous data on Trk receptor activation by Trk ligands, NT3 and BDNF (Ramekers et al., 2015; Ruel et al., 2007; Shepherd et al., 2005; Shinohara et al., 2002; Wise et al., 2005). NT3 and BDNF have long been shown to be protective or ameliorative for neural loss in the cochlea. The roles of NT3 and BDNF may be different in synaptogenesis, fiber growth, and survival. The latter roles, neuron survival and growth, are the most thoroughly characterized of the effects and are found throughout the nervous system. NT3 and BDNF do not appear to be 
equivalent for these roles (Fritzsch et al., 2006; Green et al., 2012; Tessarollo et al., 2004). Both neurotrophins are present in the inner ear, and several studies have shown that NT3 is the principal neurotrophin in the cochlea, whereas BDNF has a more important role in the vestibular system (Fritzsch et al., 1997). However, there is considerable overlap of function. Indeed, the two neurotrophins have been used together in many studies (Wise et al., 2005), and both stimulate neurite outgrowth and neuron survival.

Neurotrophins also show distinct actions in regeneration of neurons. This distinction may be key to the effects of AT on regeneration vs protection. AT has been shown to have TrkB activity with no TrkC activity (Jang et al., 2009). There are some indications that NT3 in particular is effective in cochlear hearing loss, whereas BDNF is effective in vestibular dysfunction due to synaptic lesions (Gomez-Casati et al., 2010; Suzuki et al., 2016; Wan et al., 2014). NT3 but not BDNF appeared to reverse the synaptopathy in a genetic model (Wan et al., 2014), and NT3 application to the round window (Suzuki et al., 2016) protected synapses from noise induced synaptopathy. If indeed NT3 stimulation of TrkC, but not BDNF stimulation of TrkB can repair cochlear synapses, then it seems logical that a TrkB agonist would be suboptimal. As we see a protective effect when the drug is given prior to noise exposure, as well as a regenerative effect when the drug is given after noise exposure, we believe that TrkB stimulation is effective for the stimulation of synaptogenesis in the cochlea. The effect of AT suggests that TrkB agonist activity is sufficient to restore synapses. It is hard to compare to the results on NT3 vs BDNF in transgenics where the concentration of peptide is unknown and may differ from the BDNF equivalents we added. However, we cannot rule out the 
possibility that high concentrations of the drug could also produce some activation of TrkC.

BDNF has a stimulatory effect on regeneration in many systems and is released in response to damage. In studies of aminoglycoside induced hearing loss, ears that were administered neurotrophins after the insult showed less neural loss (Ramekers et al., 2015; Shinohara et al., 2002; Wise et al., 2005); however, this effect was ascribed to protection from neural loss that occurs secondary to the loss of hair cells. Regeneration of synapses was reported in the newborn rat in vitro (Wang and Green, 2011). In that case the synapses partially recovered spontaneously, and the recovery could be augmented by BDNF or NT3. However, the endogenous factor responsible for the spontaneous regeneration appeared to be NT3. Protection of hearing from noise exposure was correlated to secretion of BDNF that occurred at night but not during the day (Meltser et al., 2014). In the studies reported here, administration of AT after the noise exposure restored synapses when it was at sufficient concentrations. Posterior semicircular canal delivery proved the most effective route and was presumed to be successful because drug reached the cochlear perilymph at sufficient concentrations. Given the regenerative effect of AT on cochlear neurons, the possibility remains that the observed protective effect was also a regenerative effect occurring after the noise exposure, i.e. a fast regeneration comprising synaptic remodeling on a rapid time scale as occurs in synaptic spines in a process driven by BDNF (Vignoli et al., 2016). The time window we looked at for administration of the drug was limited to the period shortly (2 days) after noise exposure. Development of a regenerative treatment using this drug will require assessment of the full extent of the post-damage window for a therapeutic effect. 
Tricyclic anti-depressants like AT have Trk agonist activity (Jang et al., 2009).

Interestingly, this activity was discovered long after their introduction for the treatment of mood disorders. The compounds were initially used for inhibition of serotonin and noradrenaline transport (Snyder and Peroutka, 1982; Snyder and Yamamura, 1977; U'Prichard et al., 1978). Since the discovery of the neurotrophic activity the compounds have been tested for the amelioration of various types of hearing loss (Shibata et al., 2007; Yu et al., 2012; Yu et al., 2013). The activity on a genetic hearing disorder was striking (Yu et al., 2013) and was thought to mimic BDNF activity. The effect on noise damage was thought to be due to protection of SGNs (Shibata et al., 2007). AT is a welltolerated drug that has been tested thoroughly for mood disorders, and our data suggest that AT or related drugs could have important clinical applications in noise-induced hidden hearing loss as studied here and potentially in age-related hidden hearing loss related to cochlear deafferentation.

\section{References}

Brugeaud, A., Tong, M., Luo, L., and Edge, A.S. (2014). Inhibition of repulsive guidance molecule, RGMa, increases afferent synapse formation with auditory hair cells. Dev Neurobiol 74, 457-466.

Fernandez, K.A., Guo, D., Micucci, S., De Gruttola, V., Liberman, M.C., and Kujawa, S.G. (2020). Noise-induced Cochlear Synaptopathy with and Without Sensory Cell Loss. Neuroscience 427, 43-57.

Fernandez, K.A., Jeffers, P.W., Lall, K., Liberman, M.C., and Kujawa, S.G. (2015). Aging after noise exposure: acceleration of cochlear synaptopathy in "recovered" ears. J Neurosci 35, 7509-7520.

Fritzsch, B., Beisel, K.W., and Hansen, L.A. (2006). The molecular basis of neurosensory cell formation in ear development: a blueprint for hair cell and sensory neuron regeneration? Bioessays 28, 1181-1193.

Fritzsch, B., Farinas, I., and Reichardt, L.F. (1997). Lack of neurotrophin 3 causes losses of both classes of spiral ganglion neurons in the cochlea in a region-specific fashion. $\mathrm{J}$ Neurosci 17, 6213-6225. 
Gomez-Casati, M.E., Murtie, J.C., Rio, C., Stankovic, K., Liberman, M.C., and Corfas, G. (2010). Nonneuronal cells regulate synapse formation in the vestibular sensory epithelium via erbB-dependent BDNF expression. Proc Natl Acad Sci U S A 107, 1700517010.

Green, S.H., Bailey, E., Wang, Q., and Davis, R.L. (2012). The Trk A, B, C's of neurotrophins in the cochlea. Anat Rec (Hoboken) 295, 1877-1895. Jang, S.W., Liu, X., Chan, C.B., Weinshenker, D., Hall, R.A., Xiao, G., and Ye, K. (2009). Amitriptyline is a TrkA and TrkB receptor agonist that promotes TrkA/TrkB heterodimerization and has potent neurotrophic activity. Chem Biol 16, 644-656. Kujawa, S.G., and Liberman, M.C. (2006). Acceleration of age-related hearing loss by early noise exposure: evidence of a misspent youth. J Neurosci 26, 2115-2123. Kujawa, S.G., and Liberman, M.C. (2009). Adding insult to injury: cochlear nerve degeneration after "temporary" noise-induced hearing loss. J Neurosci 29, 14077-14085. Kujawa, S.G., and Liberman, M.C. (2015). Synaptopathy in the noise-exposed and aging cochlea: Primary neural degeneration in acquired sensorineural hearing loss. Hear Res 330, 191-199.

Martinez-Monedero, R., Corrales, C.E., Cuajungco, M.P., Heller, S., and Edge, A.S. (2006). Reinnervation of hair cells by auditory neurons after selective removal of spiral ganglion neurons. J Neurobiol 66, 319-331.

Meltser, I., Cederroth, C.R., Basinou, V., Savelyev, S., Lundkvist, G.S., and Canlon, B. (2014). TrkB-mediated protection against circadian sensitivity to noise trauma in the murine cochlea. Curr Biol 24, 658-663.

Muller, M., von Hunerbein, K., Hoidis, S., and Smolders, J.W. (2005). A physiological place-frequency map of the cochlea in the CBA/J mouse. Hear Res 202, 63-73.

Nadol, J.B., Jr. (1997). Patterns of neural degeneration in the human cochlea and auditory nerve: implications for cochlear implantation. Otolaryngol Head Neck Surg 117, 220228.

Parker, M., Brugeaud, A., and Edge, A.S. (2010). Primary culture and plasmid electroporation of the murine organ of Corti. J Vis Exp.

Poduslo, J.F., and Curran, G.L. (1996). Permeability at the blood-brain and blood-nerve barriers of the neurotrophic factors: NGF, CNTF, NT-3, BDNF. Brain research Molecular brain research 36, 280-286.

Pujol, R., Lenoir, M., Robertson, D., Eybalin, M., and Johnstone, B.M. (1985). Kainic acid selectively alters auditory dendrites connected with cochlear inner hair cells. Hear Res 18, 145-151.

Pujol, R., and Puel, J.L. (1999). Excitotoxicity, synaptic repair, and functional recovery in the mammalian cochlea: a review of recent findings. Ann N Y Acad Sci 884, 249-254. Ramekers, D., Versnel, H., Strahl, S.B., Klis, S.F., and Grolman, W. (2015). Temporary Neurotrophin Treatment Prevents Deafness-Induced Auditory Nerve Degeneration and Preserves Function. J Neurosci 35, 12331-12345.

Ruel, J., Wang, J., Rebillard, G., Eybalin, M., Lloyd, R., Pujol, R., and Puel, J.L. (2007). Physiology, pharmacology and plasticity at the inner hair cell synaptic complex. Hear Res 227, 19-27.

Sergeyenko, Y., Lall, K., Liberman, M.C., and Kujawa, S.G. (2013). Age-related cochlear synaptopathy: an early-onset contributor to auditory functional decline. J Neurosci 33, 13686-13694. 
Shepherd, R.K., Coco, A., Epp, S.B., and Crook, J.M. (2005). Chronic depolarization enhances the trophic effects of brain-derived neurotrophic factor in rescuing auditory neurons following a sensorineural hearing loss. J Comp Neurol 486, 145-158.

Shibata, S.B., Osumi, Y., Yagi, M., Kanda, S., Kawamoto, K., Kuriyama, H., Nishiyama, T., and Yamashita, T. (2007). Administration of amitriptyline attenuates noise-induced hearing loss via glial cell line-derived neurotrophic factor (GDNF) induction. Brain Res 1144, 74-81.

Shinohara, T., Bredberg, G., Ulfendahl, M., Pyykko, I., Olivius, N.P., Kaksonen, R., Lindstrom, B., Altschuler, R., and Miller, J.M. (2002). Neurotrophic factor intervention restores auditory function in deafened animals. Proc Natl Acad Sci U S A 99, 1657-1660. Snyder, S.H., and Peroutka, S.J. (1982). A possible role of serotonin receptors in antidepressant drug action. Pharmacopsychiatria 15, 131-134.

Snyder, S.H., and Yamamura, H.I. (1977). Antidepressants and the muscarinic acetylcholine receptor. Arch Gen Psychiatry 34, 236-239.

Suzuki, J., Corfas, G., and Liberman, M.C. (2016). Round-window delivery of neurotrophin 3 regenerates cochlear synapses after acoustic overexposure. Sci Rep 6, 24907.

Tessarollo, L., Coppola, V., and Fritzsch, B. (2004). NT-3 replacement with brainderived neurotrophic factor redirects vestibular nerve fibers to the cochlea. J Neurosci 24 , 2575-2584.

Tong, M., Brugeaud, A., and Edge, A.S. (2013). Regenerated synapses between postnatal hair cells and auditory neurons. J Assoc Res Otolaryngol 14, 321-329.

U'Prichard, D.C., Greenberg, D.A., Sheehan, P.P., and Snyder, S.H. (1978). Tricyclic antidepressants: therapeutic properties and affinity for alpha-noradrenergic receptor binding sites in the brain. Science 199, 197-198.

Vignoli, B., Battistini, G., Melani, R., Blum, R., Santi, S., Berardi, N., and Canossa, M. (2016). Peri-Synaptic Glia Recycles Brain-Derived Neurotrophic Factor for LTP

Stabilization and Memory Retention. Neuron 92, 873-887.

Wan, G., Gomez-Casati, M.E., Gigliello, A.R., Liberman, M.C., and Corfas, G. (2014).

Neurotrophin-3 regulates ribbon synapse density in the cochlea and induces synapse regeneration after acoustic trauma. Elife 3.

Wang, Q., and Green, S.H. (2011). Functional role of neurotrophin-3 in synapse regeneration by spiral ganglion neurons on inner hair cells after excitotoxic trauma in vitro. J Neurosci 31, 7938-7949.

Wang, Y., Hirose, K., and Liberman, M.C. (2002). Dynamics of noise-induced cellular injury and repair in the mouse cochlea. J Assoc Res Otolaryngol 3, 248-268.

Wise, A.K., Richardson, R., Hardman, J., Clark, G., and O'Leary, S. (2005). Resprouting and survival of guinea pig cochlear neurons in response to the administration of the neurotrophins brain-derived neurotrophic factor and neurotrophin-3. J Comp Neurol 487, 147-165.

Wong, A.C., and Ryan, A.F. (2015). Mechanisms of sensorineural cell damage, death and survival in the cochlea. Front Aging Neurosci 7, 58.

Yu, Q., Chang, Q., Liu, X., Gong, S., Ye, K., and Lin, X. (2012). 7,8,3'-

Trihydroxyflavone, a potent small molecule TrkB receptor agonist, protects spiral ganglion neurons from degeneration both in vitro and in vivo. Biochem Biophys Res Commun 422, 387-392. 
Yu, Q., Chang, Q., Liu, X., Wang, Y., Li, H., Gong, S., Ye, K., and Lin, X. (2013). Protection of spiral ganglion neurons from degeneration using small-molecule TrkB receptor agonists. J Neurosci 33, 13042-13052. 


\section{FIGURES}

Figure 1. AT acts as a TrkB agonist in SGN. (A-C) Isolated SGNs and denervated organ of Corti were co-cultured in the absence $(\mathbf{A})$, or presence $(\mathbf{B})$ of $0.5 \mu \mathrm{M}$ AT. After 6 days in culture, explants were fixed, immunostained with antibodies against neurofilament (green), CtBP2 (blue) and PSD-95 (red), and confocal images were obtained in the inner hair cell region $(\mathbf{A}, \mathbf{B})$. Juxtapositions of hair cell ribbons and afferent endings were identified by CtBP2/PSD-95 puncta (yellow arrowheads) and counted $(\mathbf{C})$, indicating a significant increase in percentage of juxtaposed CtBP2/PSD-95 puncta at inner hair cells after AT treatment. Error bars indicate mean +/- SEM; asterisk indicates $\mathrm{p}<0.05$. (D-F) Cochlear explants were cultured with (KA) or without (No KA) exposure to kainic acid $(0.4 \mathrm{mM})$ for $1 \mathrm{~h}$ followed by treatment with culture medium with $0.1 \mu \mathrm{M}$ AT $(\mathrm{KA}+\mathrm{AT})$ or $0.5 \mu \mathrm{M}$ DHF $(\mathrm{KA}+\mathrm{DHF})$. The cultures were immunostained for CtBP2 (red) and PSD-95 (green) after 48 h (D). AT (E) and DHF (F) induced significant synaptic regeneration. Error bars indicates mean $+/-$ SEM ( $n=3$ for control and 6 for AT in $\mathbf{E}$; $n=6$ for control and 6 for DHF in $\mathbf{F}$ ); asterisks indicate $\mathrm{p}<0.05$.

\section{Figure 2. Noise-induced threshold elevations were similar across groups and Trk} agonists did not alter recovery. A: Wave 1 thresholds recorded $24 \mathrm{~h}$ after noise (8-16 $\mathrm{kHz}, 100 \mathrm{~dB}$ SPL, 2h; gray bar) and before drug delivery to the posterior semicircular canal were similar across treatment groups (AT, 25mM; DHF, 5mM; AP-vehicle). Compared to strain- and age-matched, unexposed controls (Fernandez et al., 2015), maximum shifts were $\sim 40 \mathrm{~dB}$ at basal cochlear frequencies. B: By 2 wk post noise, all 
groups recovered to within $\sim 5 \mathrm{~dB}$ of baseline values. Data shown as means $\pm \mathrm{SE}, \mathrm{n}=6$ to 8/group).

Figure 3: Local, post-exposure treatment with Trk agonists recovers cochlear nerve function and IHC synapses. Animals treated with $25 \mathrm{mM}$ AT or $5 \mathrm{mM}$ DHF $48 \mathrm{~h}$ after noise showed significantly greater $A B R$ wave 1 amplitudes $\left(F_{(2,130)}=4.636, p=0.0114\right)$ $(\mathbf{A}$; shown for example at $30 \mathrm{kHz})$ and synapse counts $\left(\mathrm{F}_{(2,72)}=8.182, \mathrm{p}=0.0006\right)(\mathbf{B})$ compared to those treated with vehicle alone. Data shown as means $\pm \mathrm{SE}, \mathrm{n}=6$ to 8/group. Unexposed controls from Fernandez et al., 2015. 2-way ANOVA with Bonferroni multiple comparisons test, ${ }^{*} \mathrm{p}<0.05, *{ }^{*} \mathrm{p}<0.01$.

Figure 4. Systemic AT protection is dose-responsive. Amitriptyline (AT) is delivered systemically $(12.5,25$ or $50 \mathrm{mg} / \mathrm{kg}$ once daily for 5-9 days). Animals are noise exposed on day 3, $6 \mathrm{hr}$ after drug. In all groups, DPOAE and ABR thresholds and DPOAE amplitudes recover by 2 wks, but ABR amplitudes do not (A,B). Compared to saline vehicle controls, a significant preservation of $A B R$ wave-I amplitude $\left(\mathbf{C} ; F_{(1,392)}=70.39\right.$, $\mathrm{p}<0.0001)$ and synapse counts $\left(\mathbf{D} ; \mathrm{F}_{(1,116)}=18.90, \mathrm{p}<0.0001\right)$ at $30 \mathrm{kHz}$ is apparent in mice treated with $50 \mathrm{mg} / \mathrm{kg}$ AT. Data shown as means $\pm \mathrm{SE}, \mathrm{n}=10$ to 30/group). Unexposed controls from Fernandez et al., 2015. 2-way ANOVA with Bonferroni multiple comparisons test, $* * \mathrm{p}<0.01, * * * \mathrm{p}<0.001, * * * * \mathrm{p}<0.0001$

Figure 5. Systemic AT, delivered pre-, but not post-exposure protects cochlear nerve function and IHC synapses. Groups of animals received IP injections of AT in 
saline or saline vehicle alone according to the following treatment groups: 1) AT (12.5, 25 or $50 \mathrm{mg} / \mathrm{kg}$ ) or saline once daily for 5-9 days with noise exposure $6 \mathrm{~h}$ after the $3^{\text {rd }}$ day of treatment; 2) AT (50 mg/kg) or saline once, $6 \mathrm{~h}$ prior to noise exposure; 3) AT (50 $\mathrm{mg} / \mathrm{kg}$ ) or saline once, $3 \mathrm{~d}$ after noise exposure; 4) AT (50 mg/kg) or saline once daily for 9 days beginning 3 days after noise exposure. Together, pre-exposure AT delivery was required to achieve protection $\left(A B R\right.$ Amplitude: $F_{(2,496)}=55.49, p<0.0001$; Synapses: $\mathrm{F}(2,143)=24.19, \mathrm{p}<0.0001)$. Data shown as means $\pm \mathrm{SE}, \mathrm{n}=10$ to $30 /$ group $)$. Unexposed controls from Fernandez et al., 2015. 2-way ANOVA with Bonferroni multiple comparisons test.

Figure 6: AT protection is long-lasting. Subsets of mice were held 1 year post noise. DPOAE amplitudes remained well preserved in all groups (A). Relative to age-matched, vehicle treated controls, significant conservation of ABR wave I amplitude (B) was observed in mice treated with AT $50 \mathrm{mg} / \mathrm{kg}$ for 9 days beginning 3 days pre noise $\left(\mathrm{F}_{(1,122)}=33.88, \mathrm{p}<0.0001\right)(\mathbf{B})$. The number of synapses remaining at $2 \mathrm{wks}$ also was also maintained 1 year after noise exposure $(\mathbf{C})$, with significantly more synapses in the high frequency $32 \mathrm{kHz}$ region for mice treated with $\operatorname{AT}\left(\mathrm{F}_{(1,131)}=16.71, \mathrm{p}<0.0001\right)$. Group means \pm SEMs are shown: $n=7-30$ mice per group. Unexposed controls from Fernandez et al., 2015. 2-way ANOVA with Bonferroni multiple comparisons test, ${ }^{*} \mathrm{p}<0.05$, $* * \mathrm{p}<0.01, * * * \mathrm{p}<0.001, * * * * \mathrm{p}<0.0001$ 

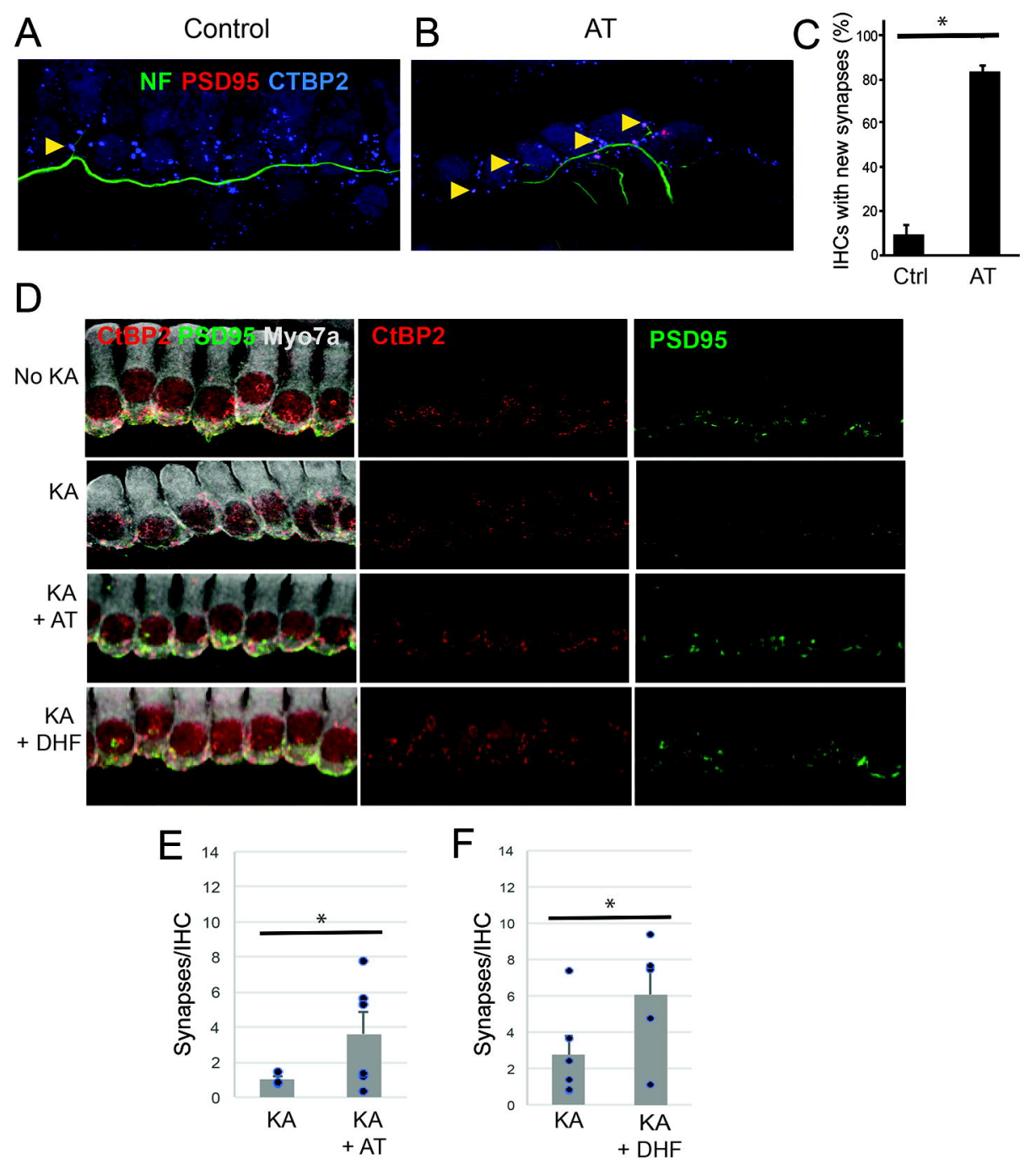


\section{4 hours}

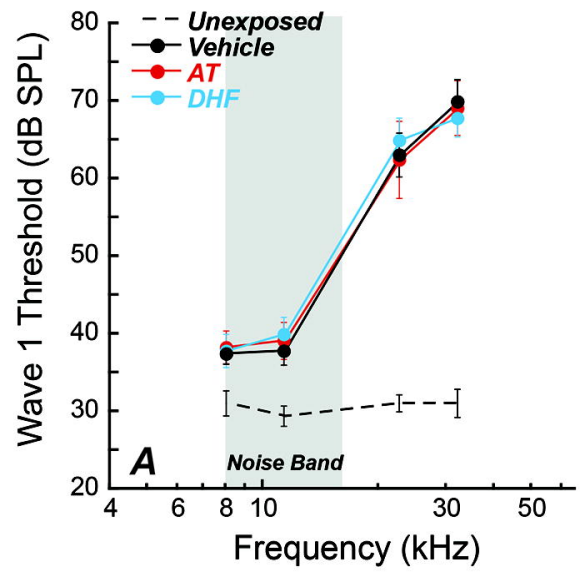

2 weeks

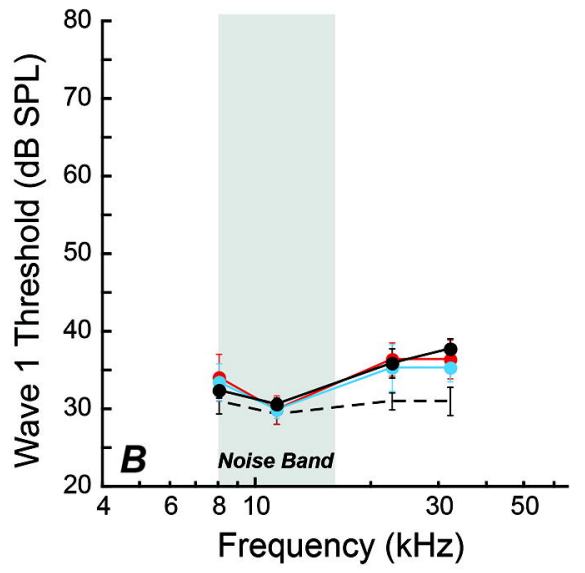




\section{ABR Wave 1}

\section{Synapses}
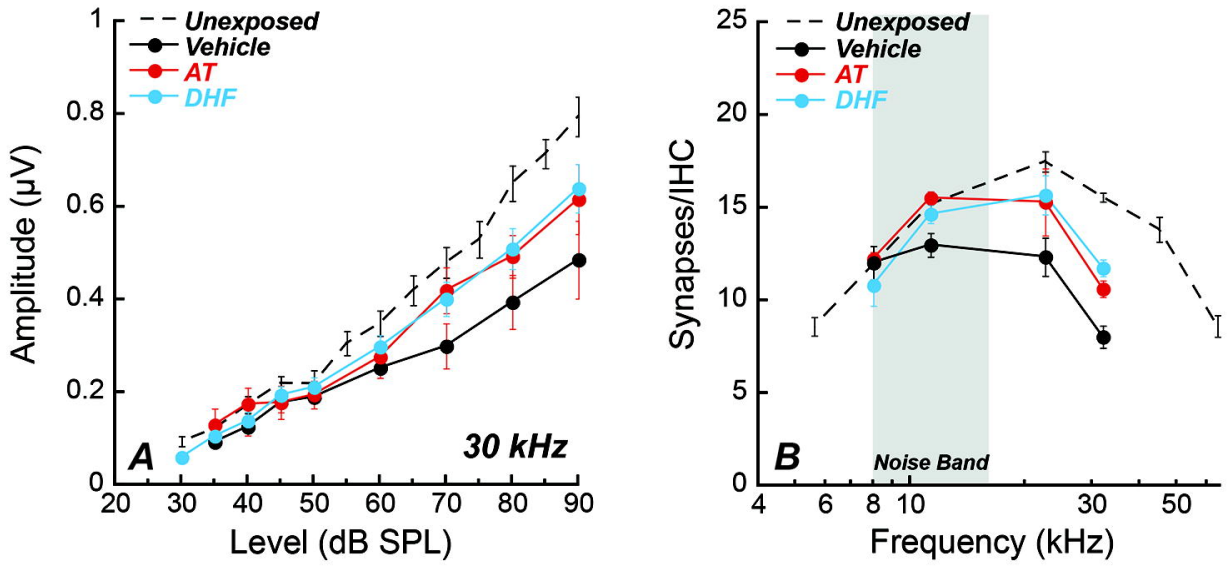


\section{DPOAE}
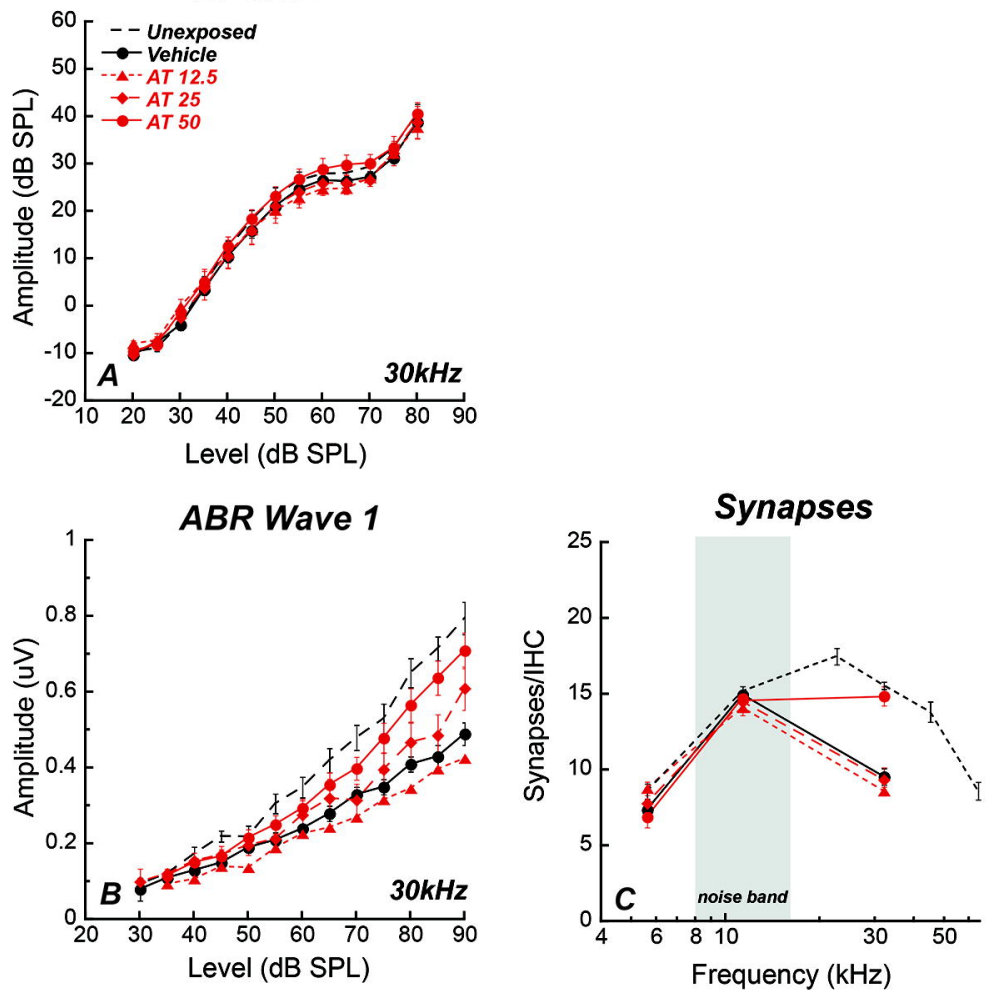


\section{ABR Wave 1}

\section{Synapses}
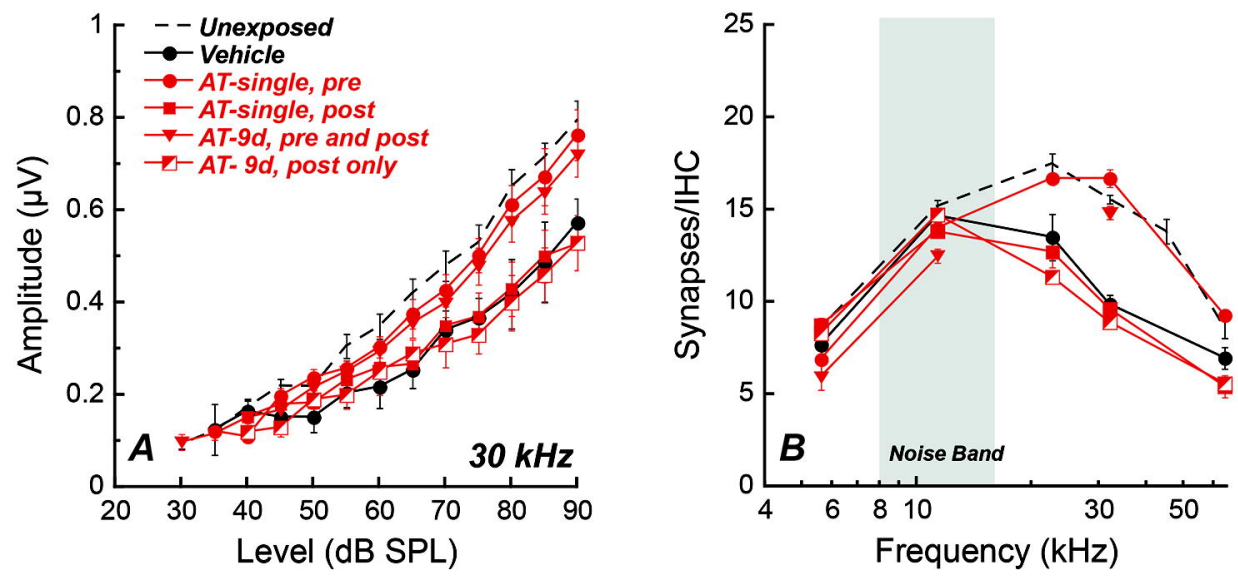


\section{DPOAE}

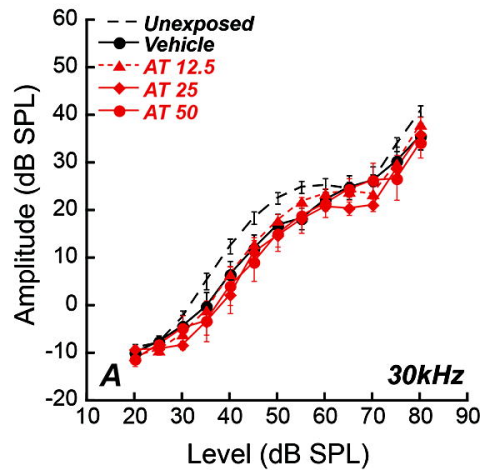

ABR Wave 1
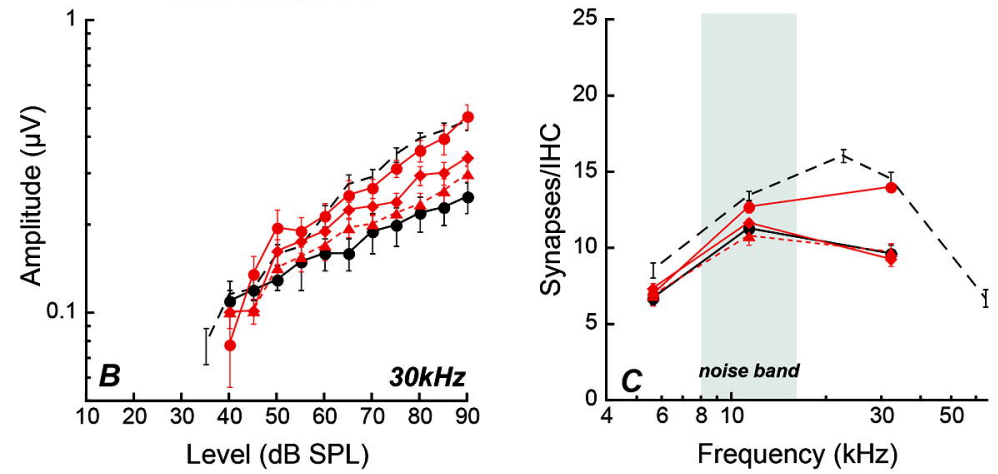